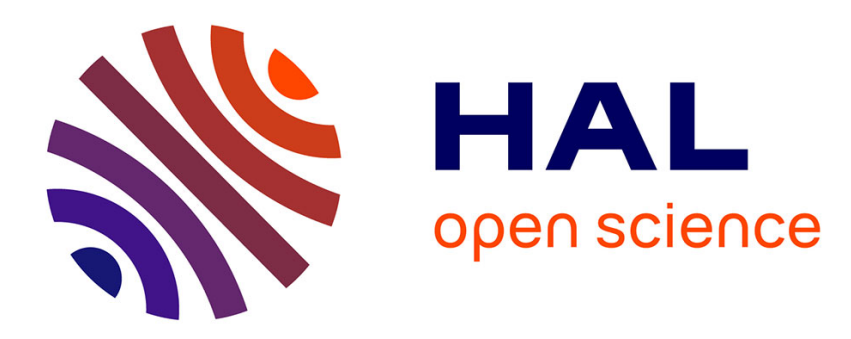

\title{
Parametric, external and self-excitation of a tower under turbulent wind flow
}

\author{
Angelo Luongo, Daniele Zulli
}

\section{To cite this version:}

Angelo Luongo, Daniele Zulli. Parametric, external and self-excitation of a tower under turbulent wind flow. Journal of Sound and Vibration, 2011, 330 (13), pp.3057-3069. hal-00788260

\section{HAL Id: hal-00788260 \\ https://hal.science/hal-00788260}

Submitted on 14 Feb 2013

HAL is a multi-disciplinary open access archive for the deposit and dissemination of scientific research documents, whether they are published or not. The documents may come from teaching and research institutions in France or abroad, or from public or private research centers.
L'archive ouverte pluridisciplinaire HAL, est destinée au dépôt et à la diffusion de documents scientifiques de niveau recherche, publiés ou non, émanant des établissements d'enseignement et de recherche français ou étrangers, des laboratoires publics ou privés. 


\title{
Parametric, external and self-excitation of a tower under turbulent wind flow
}

\author{
Angelo Luongo*, Daniele Zulli \\ DISAT - Università degli Studi dell'Aquila, 67100 L'Aquila (AQ), Italy
}

\begin{abstract}
A B S T R A C T
In this paper the analysis of a self excited tower under turbulent wind flow is carried out. The structure is considered as a one dof nonlinear system, and the implications of this modeling are deeply discussed. The stationary wind is responsible for self excitation, while the turbulent part provides both parametric and external excitations. The simultaneous presence of those excitations is taken into account in a specific resonance condition. The periodic and quasi periodic solutions are studied by means of a perturbation method and the effects of the turbulence on the dynamics of the structure are analyzed.
\end{abstract}

\section{Introduction}

Nonlinear one dof dynamical systems can experience a variety of bifurcation and instability phenomena, which can be related to different kinds of excitations. Self excited autonomous systems, under a suitable combination of parameters, undergo for instance Hopf bifurcations, in which the critical condition is reached when the total damping vanishes and, consequently, the post critical dynamics involve large amplitude oscillations. This is the case, for example, of structures subject to steady wind where the aerodynamic force introduces a damping term, opposite in sign to the structural one, depending on the wind velocity [1].

On the other hand, when dealing with non autonomous systems, the parametric or external excitation leads to a huge class of interesting phenomena such as, for example, fold bifurcations, jump phenomena, Neimark bifurcations, period doubling, and chaos $[2,3]$.

Depending on the nature of the loads, the different kinds of excitations can interact. Many articles, from different engineering fields, are dedicated to the study of interactions between external and parametric excitation in one dof systems, both from deterministic [ $\left.\begin{array}{ll}4 & 12\end{array}\right]$ and stochastic [ $\left.\begin{array}{ll}13 & 16\end{array}\right]$ points of view, while the interaction between parametric and self excitations is the object of investigations in [17 19]. The dynamics under the concurrent presence of self and mono or multi frequency external excitation are also analyzed in [2].

In some cases, for example in machine dynamics, the contemporary occurrence of the three kinds of excitations, namely self, parametric and external ones, is possible in one dof systems. In this context, the related papers are [20 23]. In particular, in $[20,21]$ comparisons among solutions of a one dof Rayleigh Duffing parametrically excited system, obtained in absence as well as in presence of external excitation, are carried out. In [22], the multiple scales method is used to obtain a law to control the amplitude of oscillations of a cantilever beam by varying the linear and nonlinear damping coefficients, when both parametric and external excitations were present. In [23], several resonance conditions are

\footnotetext{
* Corresponding author.

E-mail addresses: angelo.luongo@univaq.it (A. Luongo), daniele.zulli@univaq.it (D. Zulli).
} 
analyzed in a multi frequency forced system, giving rise to periodic, quasi periodic and chaotic motions. In [24], interesting effects of synchronization and transitions to chaotic motions were observed for these kinds of problems.

Even in the case of structures exposed to turbulent wind, the three sources of excitations can interact. Indeed the steady part of the flow is responsible for the self excitation, while the unsteady flow brings on parametric and external forces. In this framework, in [25] a one dof system, bearing the simultaneous presence of the self, parametric and external excitations, is considered to study the galloping phenomena of tall cantilever structures. There, the multiple scales method (MSM) is used to analyze the response of the system to a mono frequent unsteady wind flow, in case of primary and secondary resonances. It is shown that the unsteady component can cause a significant decrease in the wind speed at which large amplitude oscillations occur; moreover, the contribution of the unsteady component is less prominent at high wind velocities, where the amplitude of galloping oscillations is very similar to the case of steady wind flow.

This paper aims to extend the analysis, carried out in [25], of self excited structures under turbulent wind. The objective is to take into account the possible occurrence of Hopf bifurcations, obtained by varying the steady part of the wind, and to analyze the modifications on the solutions due to the turbulent part, which herein is considered multi frequent. To this end a tower, under the simultaneous presence of the self, parametric and external excitations, is considered. The tower is a linear elastic multistory shear type frame, modeled through a corresponding homogeneous scheme of continuous shear cantilever, and then reduced to a one dof system via a modal representation. The order of magnitude of the error introduced by these approximations is discussed with reference to a specific example. The nonlinear terms appearing in the system are provided by the aeroelastic force, while the nonlinear elastic terms are considered negligible. The multiple scale method [2] is applied, under a specific resonance condition. A set of amplitude modulation equations (AME) is obtained, describing the slow dynamics of the system in terms of amplitude of oscillations and phase difference. A bifurcation analysis is carried out and the equilibrium solutions, representing periodic oscillations, are analyzed in the space of the bifurcation parameters. Their stability is discussed, highlighting the influence, on the response of the structure, of the three components of the excitations in different regions of the parameter space. In particular, as a consequence of the multi frequency nature of the turbulence, the excitations can act separately. The analysis is also extended to quasi periodic solutions.

\section{The model}

A tower of square section, constituted by a multistory shear type frame, is subjected to unsteady wind flow, uniformly distributed all along its height. The dynamics of the structure are studied in the across wind plane (see Fig. 1). The along wind forces modify the equilibrium position of the tower, which becomes curvilinear; this effect is small and is typically considered as an imperfection in the model (see [26]). However, in this paper the along wind dynamics are neglected.

In Fig. $1, v_{i}(t)$ is the (time dependent) cross wind transversal displacement of the $i$ th story of the tower. The inter story height of the tower is $h$, and the dimensions of the cross section are $b \times b$. The total height of the tower is $\ell$.

\subsection{Homogeneous continuous model}

A corresponding homogeneous scheme, constituted by a planar shear rod with square cross section, is introduced (see Fig. 2). The rod is clamped at one end and free at the other end. Kinematics are assumed linear, according to the fact that cantilever beams exhibit a weak nonlinear behavior (see [27]).

The shear rod is a one dimensional polar continuum, constituted by an axis line, whose transversal displacement is indicated as $v(s, t)$ and by cross sections which remain planar and are unable to rotate: $\varphi(s, t) \equiv 0$. The only non null strain measure is the shear strain $\gamma(s, t):=v^{\prime}(s, t)$, where the prime denotes differentiation with respect to the abscissa $s$, while the curvature is identically null: $\kappa(s, t):=\varphi^{\prime}(s, t) \equiv 0$. The stress at the generic cross section is described by the shear force $T(s, t)$ and the bending moment $M(s, t)$ and, if $p(s, t)$ is the linear density of external in plane load (including the inertia forces), the balance reads as

$$
\begin{aligned}
T^{\prime}(s, t)+p(s, t) & =0 \\
M^{\prime}(s, t)+T(s, t) & =0
\end{aligned}
$$

In Eq. (1), the bending moment is reactive, while the shear force is active. Assuming a linear homogeneous elastic material, it holds that $T(s, t)=G A \gamma(s, t)$, where $G A$ is the shear stiffness of the rod, assumed constant. In this way, Eq. $\left(1_{1}\right)$ becomes

$$
G A v^{\prime \prime} \quad m v+p_{n i}=0
$$

where the contribution of the inertia force is introduced through the linear mass density $m$, the dot stands for time derivative and $p_{n i}$ is the non inertial contribution to the load. Once known $v(s, t)$, one can get back to the bending moment 
(a)

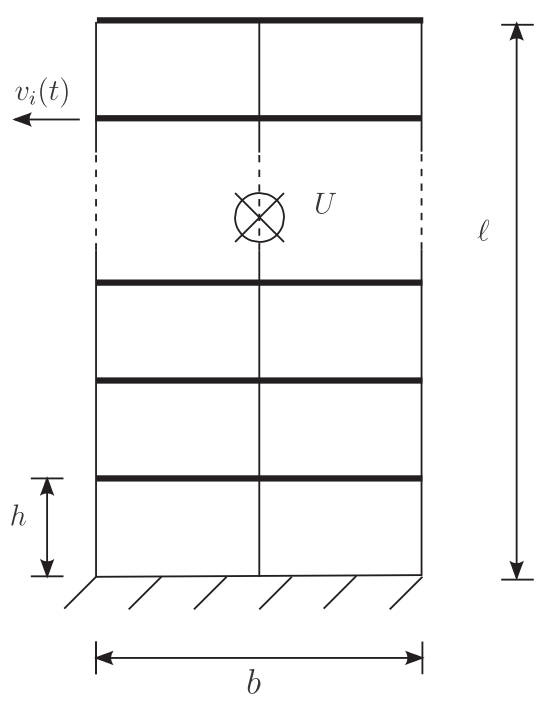

(b)

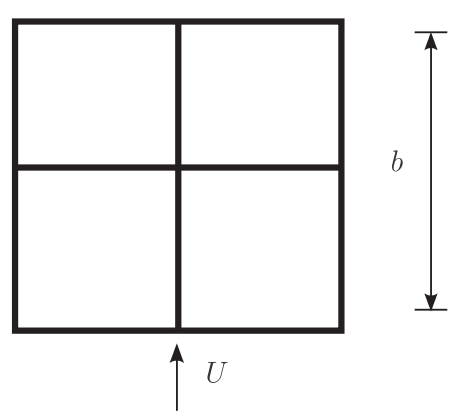

Fig. 1. Frontal (a) and top (b) views of the tower subjected to the wind flow uniformly distributed all along its height.

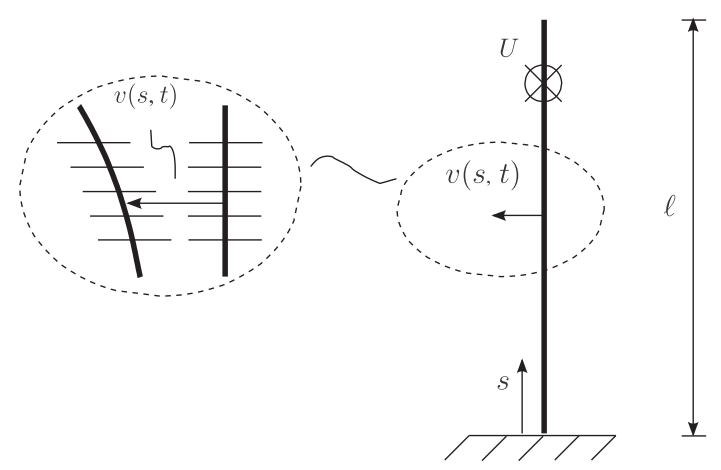

Fig. 2. Frontal view of the homogeneous model.

by integrating Eq. $\left(1_{2}\right)$, namely

$$
M(s, t)=M(0, t) \quad \int_{0}^{s} G A v^{\prime}(\zeta, t) \mathrm{d} \zeta=M(0, t) \quad G A v(s, t)
$$

The value of the homogeneous shear stiffness is identified by equating the strain energy density of the discrete and the homogeneous models: it holds that $G A=n_{c} 12 E I / h^{2}$, where $n_{c}$ is the number of columns in the generic story and $E I$ is the bending stiffness of each column, assumed the same throughout the tower. 
The boundary conditions read as

$$
v(0, t)=0
$$

$$
G A v^{\prime}(\ell, t)=0
$$

The non inertial contribution to the load in Eq. (2) can be decomposed as

$$
p_{n i}=p_{d}+p_{a}
$$

where $p_{d}$ and $p_{a}$ are the damping and the aerodynamic parts, respectively. In particular $p_{d}$ contains both internal (Kelvin model) and external (drag) damping and reads as

$$
p_{d}=\eta \dot{v}^{\prime \prime}(s, t) \quad c \dot{v}(s, t) \quad \eta \dot{v}^{\prime}(\ell, t) \delta(s \quad \ell)
$$

where $\eta$ and $c$ are the internal and external viscous damping coefficients, respectively. Such a damping force is consistent with the Rayleigh damping model used for discrete systems.

The aerodynamic part of Eq. (5) is related to the wind which blows orthogonally to the beam axis with time dependent velocity $U(t)$. It triggers a lift force on the rod lying on the plane of the structure (see [28]), of type

$$
p_{a}=\frac{1}{2} \rho U^{2} b\left[\mathcal{A}_{0}+\mathcal{A}_{1}\left(\frac{\dot{v}}{U}\right)+\mathcal{A}_{2}\left(\frac{\dot{v}}{U}\right)^{2}+\mathcal{A}_{3}\left(\frac{\dot{v}}{U}\right)^{3}\right]
$$

where $\mathcal{A}_{j}(j=1,2,3)$ are the aerodynamic coefficients relevant to square sections, $\rho$ the air mass density, and terms up to power three have been considered. The wind velocity can be decomposed as $U(t)=\bar{U}+u(t)$, where $\bar{U}$ is a constant (average) part, representing the steady component, and $u(t)$ is a periodically time dependent part, representing the turbulence. In particular, considering the turbulent part small compared to the steady one and expanding with Taylor's polynomial, the lift force in Eq. (7) turns out to be

$$
p_{a}=\frac{1}{2} \rho b\left[c_{0}+c_{1} \dot{v}+c_{2} \dot{v}^{2}+c_{3} \dot{v}^{3}\right]
$$

where $c_{0}=\mathcal{A}_{0}\left(\bar{U}^{2}+2 \bar{U} u(t)\right), \quad c_{1}=\mathcal{A}_{1}(\bar{U}+u(t)), \quad c_{2}=\mathcal{A}_{2}$, and $c_{3}=\mathcal{A}_{3}\left(1 / \bar{U} u(t) / \bar{U}^{2}\right)$. Therefore, the aerodynamic force provides, by means of its steady part, terms which can be responsible for galloping and, by means of its turbulent part, time dependent terms, assumed periodic. Here a slight difference is present with respect to the aerodynamic force used in [25], where also the quadratic coefficient $c_{2}$ was assumed to be time dependent, inconsistently with Eq. (7).

Non dimensional quantities are introduced by letting

$$
s^{*}=\frac{s}{\ell}, \quad t^{*}=\omega t, \quad v^{*}=\frac{v}{\ell}, \quad \bar{U}^{*}=\frac{\bar{U}}{\bar{U}_{c}}, \quad u^{*}=\frac{u}{\bar{U}_{c}}
$$

where $\omega$ is the first natural frequency of the beam and $\bar{U}_{c}$ is the critical value of the wind velocity, to be determined later. For convenience of notation, the star will be dropped ahead.

\subsection{Discrete model}

The continuous system (2) (4) is discretized as a one dof system via the Galerkin method, assuming as trial function the first mode of the rod, evaluated in the absence of wind and damping, namely $v(s, t)=x(t) \phi(s)$, where

$$
\phi(s)=\sin \left(\frac{\pi}{2} s\right)
$$

Possible internal resonances with higher modes are excluded.

The resulting non dimensional, second order, non homogeneous, time periodic, ordinary differential equation is

$$
x+\left(c_{a}(1 \quad \bar{U}) \quad b_{1} u(t)\right) \dot{x}+x+b_{2} \dot{x}^{2}+\left(\frac{b_{31}}{\bar{U}}+\frac{b_{32}}{\bar{U}^{2}} u(t)\right) \dot{x}^{3}=\eta_{1} \bar{U} u(t)+\eta_{2} \bar{U}^{2}
$$

where the dot denotes differentiation with respect to the non dimensional time $t$. It contains elastic, viscous and inertial linear terms, but also quadratic and cubic terms in the velocity, generated by the aerodynamic forces. The expressions of the coefficients in Eq. (11) are reported in Appendix A, together with the dimensional critical velocity $\bar{U}_{c}$, obtained by vanishing the total (structural plus aerodynamic) linear damping. The critical condition (Hopf bifurcation) therefore occurs at the non dimensional speed $\bar{U}=1$, according to Eq. $\left(9_{4}\right)$.

The turbulent part is considered as periodic and constituted of its first two frequencies: $u(t)=u_{1} \sin (\Omega t)+u_{2} \sin (2 \Omega t)$, where $u_{1}$ and $u_{2}$ are two amplitudes and $\Omega$ is the fundamental frequency, whose critical value is $\Omega_{c}=1$. In this way, both external and parametric excitations are resonant. The wind velocity $\bar{U}$ and the forcing frequency $\Omega$ are taken as bifurcation parameters, both having unitary critical values. The objective is to study the behavior of the system in the neighborhood of the critical condition in the space of the bifurcation parameters. 


\section{The multiple scale analysis}

A dimensionless small parameter $\varepsilon$ is introduced and the increments of the critical parameters are defined as

$$
\begin{aligned}
& \bar{U}=1+\varepsilon V \\
& \Omega=1+\varepsilon \sigma
\end{aligned}
$$

moreover the dependent variable $x$ and the coefficients of Eq. (11) are rescaled as follows: $x=\varepsilon^{1 / 2} \hat{x} ; b_{1}=\varepsilon \hat{b}_{1} ; b_{2}=\varepsilon^{1 / 2} \hat{b}_{2}$; $\left\{\eta_{1}, \eta_{2}\right\}=\varepsilon^{3 / 2}\left\{\hat{\eta}_{1}, \hat{\eta}_{2}\right\}$. The other coefficients are of order 1 . It means that the linear damping is assumed small and the coefficients of the external and parametric excitation, as well as the nonlinear terms, are ordered so that they appear at the highest order perturbation equations considered here.

\subsection{Perturbation equations}

To apply the multiple scales method, the dependent variable is expanded as

$$
\hat{x}=\hat{x}_{0}+\varepsilon \hat{x}_{1}+\cdots
$$

After introducing two independent time scales $t_{0}:=t$ and $t_{1}:=\varepsilon t$, the derivative with respect to the time assumes the expression $\mathrm{d} / \mathrm{d} t=d_{0}+\varepsilon d_{1}$, where $d_{i}:=\partial / \partial t_{i}$. As a consequence, the perturbation equations, divided by $\varepsilon^{1 / 2}$, read (omitting the hats) as

$$
\begin{aligned}
& \mathcal{O}\left(\varepsilon^{0}\right): d_{0}^{2} x_{0}+x_{0}=0 \\
& \mathcal{O}\left(\varepsilon^{1}\right): d_{0}^{2} x_{1}+x_{1}=2 d_{0} d_{1} x_{0}+\left(c_{a} V+b_{1} u\left(t_{0}\right)\right) d_{0} x_{0} \quad b_{2}\left(d_{0} x_{0}\right)^{2} \quad\left(b_{31}+b_{32} u\left(t_{0}\right)\right)\left(d_{0} x_{0}\right)^{3}+\eta_{1} u\left(t_{0}\right)+\eta_{2}
\end{aligned}
$$

Eq. $\left(14_{1}\right)$ admits the following solution:

$$
x_{0}=A\left(t_{1}\right) \exp \left(i t_{0}\right)+c c
$$

where $c c$ denotes the complex conjugate, $i$ is the imaginary unit and $A$ is an unknown complex amplitude. Using Eq. (12 $)$, the turbulent part is written as

$$
u\left(t_{0}, t_{1}\right)=u_{1} \exp \left(i(1+\varepsilon \sigma) t_{0}\right)+u_{2} \exp \left(2 i(1+\varepsilon \sigma) t_{0}\right)+c c
$$

By substituting Eqs. (15) and (16) in Eq. $\left(14_{2}\right)$ and by zeroing the resonant terms which arise on the right hand side, a differential equation in $A$ is obtained. Hence, by coming back to the true time $t$ and reabsorbing $\varepsilon$, this equation constitutes the amplitude modulation equation (AME). It reads as

$$
\dot{A}=\frac{1}{2} c_{a} V A \quad \frac{3}{2} b_{31} A^{2} \bar{A} \quad \frac{\eta_{1} u_{1}}{4} e^{i \sigma t}+i \frac{b_{1}}{4} u_{2} \bar{A} e^{2 i \sigma t}+i \frac{b_{32}}{4} u_{2} A^{3} e^{-2 i \sigma t} \quad i \frac{3}{4} b_{32} u_{2} A \bar{A}^{2} e^{2 i \sigma t}
$$

It is worth noting how the turbulent component provides external excitation by its term of amplitude $u_{1}$ and parametric excitation (both linear and cubic), by its term of amplitude $u_{2}$.

The polar form of Eq. (17), obtained by letting $A:=\frac{1}{2} a e^{i \vartheta}$ and $\varphi:=\sigma t \quad \vartheta$, is

$$
\begin{gathered}
\dot{a}=\frac{1}{2}\left(c_{a} V \frac{1}{4} b_{1} u_{2} \sin (2 \varphi)\right) a+\left(\frac{3}{8} b_{31}+\frac{b_{32}}{4} u_{2} \sin (2 \varphi)\right) a^{3} \quad \frac{\eta_{1}}{2} u_{1} \cos \varphi \\
a \dot{\varphi}=\left(\sigma \quad \frac{b_{1}}{4} u_{2} \cos (2 \varphi)\right) a+\frac{b_{32}}{8} u_{2} a^{3} \cos (2 \varphi)+\frac{\eta_{1}}{2} u_{1} \sin \varphi
\end{gathered}
$$

\subsection{Fixed points analysis}

Fixed points of Eq. (18), obtained requiring $\dot{a}=\dot{\varphi}=0$, represent periodic oscillations of the rod. Here, analytical expressions for them are sought.

In the absence of turbulence $\left(u_{1}=u_{2}=0\right)$, the simple Hopf bifurcation case is obtained. Just Eq. $\left(18_{1}\right)$ is interesting, the phase $\varphi$ being a slave variable. In this case, besides the trivial solution $a=0$, the self excitation is responsible for galloping, and the classical bifurcated solution occurs. That is the following:

$$
a=2 \sqrt{\frac{c_{a} V}{3 b_{31}}}
$$

which (when $c_{a}>0$ ) occurs for $V \geq 0$. It is always stable and indicates periodic oscillations of the rod.

Turbulence will be addressed considering first the sole external contribution $\left(u_{1} \neq 0, u_{2}=0\right)$, then considering the sole parametric contribution $\left(u_{1}=0, u_{2} \neq 0\right)$ and finally considering the two contributions together $\left(u_{1} \neq 0, u_{2} \neq 0\right)$. In all these cases, the relevant equations are both Eqs. $(18)_{12}$. 
In the first case $\left(u_{1} \neq 0, u_{2}=0\right)$, two nonlinear algebraic equations can be drawn in the following way: $\cos \varphi$ is obtained by zeroing the right hand side of Eq. $\left(18_{1}\right), \sin \varphi$ is obtained by zeroing the right hand side of Eq. $\left(18_{2}\right)$, and then the variable $\varphi$ is condensed using the relation $\cos ^{2} \varphi+\sin ^{2} \varphi=1$. The resulting equation, where only $a$ appears as unknown, is the following:

$$
\frac{a^{2}}{16 \eta_{1}^{2} u_{1}^{2}}\left(3 a^{2} b_{31}\left(3 a^{2} b_{31} \quad 8 c_{a} V\right)+16\left(c_{a}^{2} V^{2}+4 \sigma^{2}\right)\right)=1
$$

Eq. (20) is bi quadratic, and its roots can be obtained analytically (not reported here for brevity). The trivial solution does not exist in this case.

In the second case $\left(u_{1}=0, u_{2} \neq 0\right)$, the corresponding two nonlinear algebraic equations can be obtained as follows: $\sin (2 \varphi)$ is obtained by zeroing the right hand side of Eq. $\left(18_{1}\right), \cos (2 \varphi)$ is obtained by zeroing the right hand side of Eq. $\left(18_{2}\right)$, and then, again, the variable $\varphi$ is condensed using the relation $\cos ^{2}(2 \varphi)+\sin ^{2}(2 \varphi)=1$. The resulting equation, where only $a$ appears as unknown, is the following:

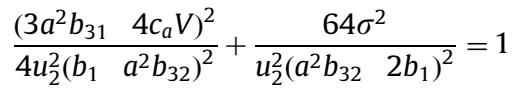

As in the previous case, Eq. (21) is bi quadratic, and its roots can be obtained analytically. The trivial solution does exist in this case.

In the third case $\left(u_{1} \neq 0, u_{2} \neq 0\right)$, it is not possible to apply a corresponding procedure, since terms of type $\sin 2 \varphi, \cos 2 \varphi$, $\sin \varphi, \cos \varphi$, are concurrently present. It means that, in this case, it is necessary to solve a nonlinear system of two equations in $\varphi$ and $a$. The trivial solution does not exist in this case.

Solutions coming from Eqs. (20), (21) and from the generic case are discussed in the following section, and their stability analyzed.

\section{Numerical results}

The following numerical values are used for a case study: the height of the tower is $\ell=36 \mathrm{~m}$; the cross section is $b=16 \mathrm{~m}$ wide, the total stiffness of the single story is $E I=115318000 \mathrm{Nm}^{2}$, the mass longitudinal density is $m=4737 \mathrm{~kg} / \mathrm{m}$, the damping ratio is $\xi=0.5$ percent (corresponding to $\eta=128513 \mathrm{Ns}, c=34.8675 \mathrm{Ns} / \mathrm{m}^{2}$ in Eq. (A.3)). The inter story height is assumed $h=4 \mathrm{~m}$. The aerodynamic coefficients $\mathcal{A}_{i}, i=0, \ldots, 3$ are taken from [25] for the squared cross section: $\mathcal{A}_{0}=0.0297, \mathcal{A}_{1}=0.9298, \mathcal{A}_{2}=0.2400, \mathcal{A}_{3}=7.6770$. The air mass density is $\rho=1.25 \mathrm{~kg} / \mathrm{m}^{3}$. The (dimensional) natural frequency of the rod is $\omega=5.89 \mathrm{rad} / \mathrm{s}$. The (dimensional) critical wind velocity assumes the value $\bar{U}_{c}=30 \mathrm{~m} / \mathrm{s}$. The equation of motion (11) becomes

$$
x+(0.01 \quad 0.01(1+V+u(t))) \dot{x}+0.0155 \dot{x}^{2}+3.099(1 \quad V \quad u(t)) \dot{x}^{3}+x=5.74 \times 10^{-5}(1+2 V+2 u(t))
$$

The numerical values assumed by the coefficients appear to be consistent with the ordering performed in the perturbation analysis. A discussion on the validity of the approximations introduced in modeling this system is reported in Appendix B.

In the absence of turbulence $\left(u_{1}=u_{2}=0\right)$, the behavior of the system is described in Fig. 3 where the steady value of the amplitude is shown in terms of the increment of the mean wind velocity (Eq. (19)). The trivial solution exists everywhere. The branch of the stable bifurcated solution emanates from the bifurcation point $B_{1}$, where the trivial solution changes its stability. In correspondence with this branch, the tower experiences periodic oscillations in the across wind direction, while, for sub critical values of the wind velocity, the tower is stationary.

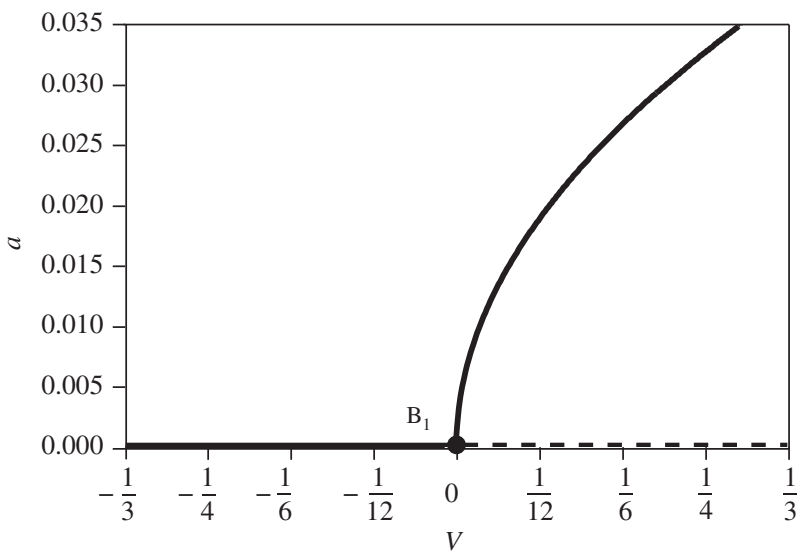

Fig. 3. Equilibrium branches in absence of turbulent wind. Continuous line: stable; dashed line: unstable. 
When the external excitation is considered at the resonant value $\left(u_{1} \neq 0, u_{2}=0\right)$, the simple galloping is modified according to Fig. 4: the trivial solution does not exist anymore, and the bifurcated solution changes as in the presence of a slight imperfection on a pitchfork bifurcation. The (perfect) solution obtained in the absence of external excitation is superimposed there to highlight that it behaves like a backbone (indicated in black in Fig. 4) for the imperfect solution. In particular, the backbone splits into two separate solutions, one on the left, the other on the right, with different stability properties. For all the possible values of $u_{1}$, the two solutions do not merge. Due to the external excitations, no rest conditions exist, so that the tower oscillates periodically for any $V$, with amplitudes provided by the stable branches.

If $V$ is kept fixed and $\sigma$ is varied, the amplitude of oscillations behave as shown in Fig. 5, where different values of $V$ are considered. For increasing values of $V$, the amplitude first increases, then the curve forms an island. On the left and right of the equilibrium branches, periodic solutions are found (shown as vertical lines corresponding to the amplitude interval of the limit cycle), which represent quasi periodic oscillations of the tower. They are coherent with the results reported in [21]. The periodic solutions disappear as a consequence of homoclinic bifurcations, occurring when the limit cycles, with period tending to infinite, touch the equilibrium branches.

A three dimensional plot of the amplitude of the motion as functions of $V$ and $\sigma$ is shown in Fig. 6 . It is quite evident how the solution forms a sort of tube around the (not shown) perfect solution. Outside the tube, at its left and right, periodic solution in terms of amplitude $a$ are found.

When the system is parametric excited $\left(u_{1}=0, u_{2} \neq 0\right)$, the amplitude $a$ depends on the velocity $V$ as shown in Fig. 7. In addition to the trivial solution, the (perfect) solution, relevant to the no turbulence case, also appears, but duplicated and translated in opposite directions. Translation on its left (right) resembles a reduction (increase) of structural damping in a Hopf bifurcation (or, in turn, of stiffness in a pitchfork bifurcation, as it occurs in the AME). In particular, the left branch is stable and the right branch is unstable. As a result of the turbulence, the value of $V$ at which galloping occurs is decreased.

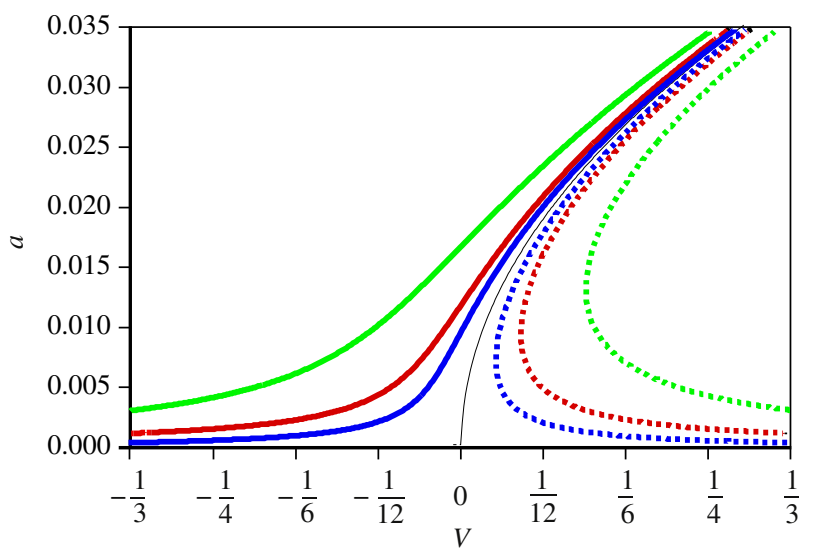

Fig. 4. Equilibrium branches in presence of turbulent wind when $u_{2}=0, \sigma \quad 0$. Black line: absence of turbulence; blue line: $u_{1}=0.017$; red line: $u_{1}=0.033$; green line: $u_{1}=0.10$. Continuous line: stable; dashed line: unstable. (For interpretation of the references to color in this figure legend, the reader is referred to the web version of this article.)

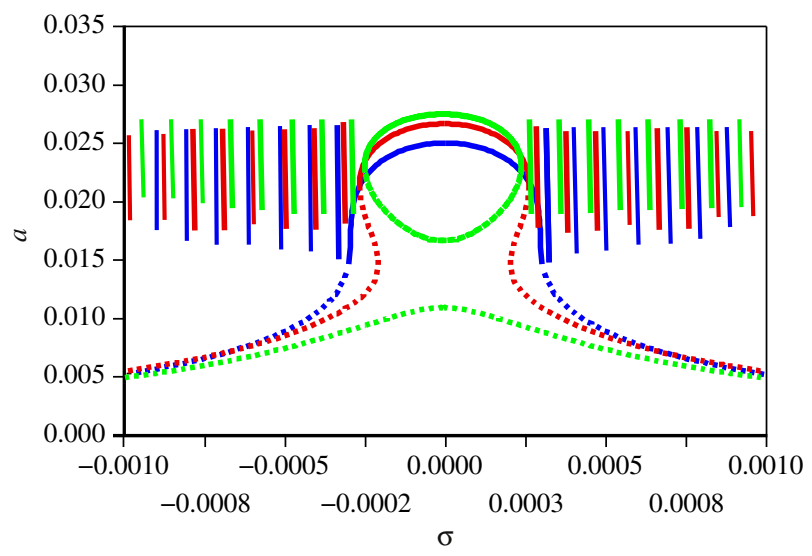

Fig. 5. Equilibrium branches in presence of turbulent wind when $u_{1}=0.033, u_{2}=0$. Blue line: $V=0.10$; red line: $V=0.117 ;$ green line: $V=0.133 \mathrm{~m} / \mathrm{s}$. Continuous line: stable; dashed line: unstable. (For interpretation of the references to color in this figure legend, the reader is referred to the web version of this article.) 


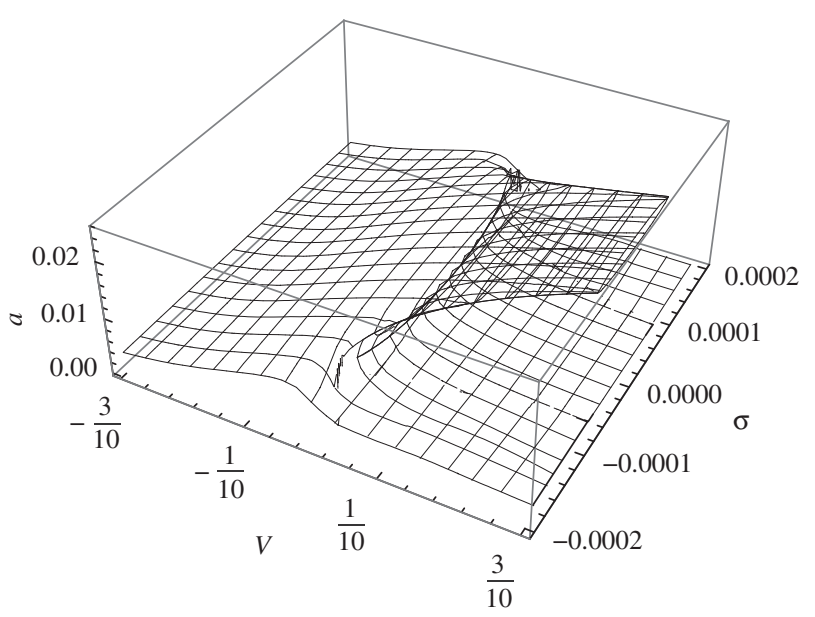

Fig. 6. Equilibrium branch in presence of external excitation $\left(u_{1}=0.033, u_{2}=0\right)$.

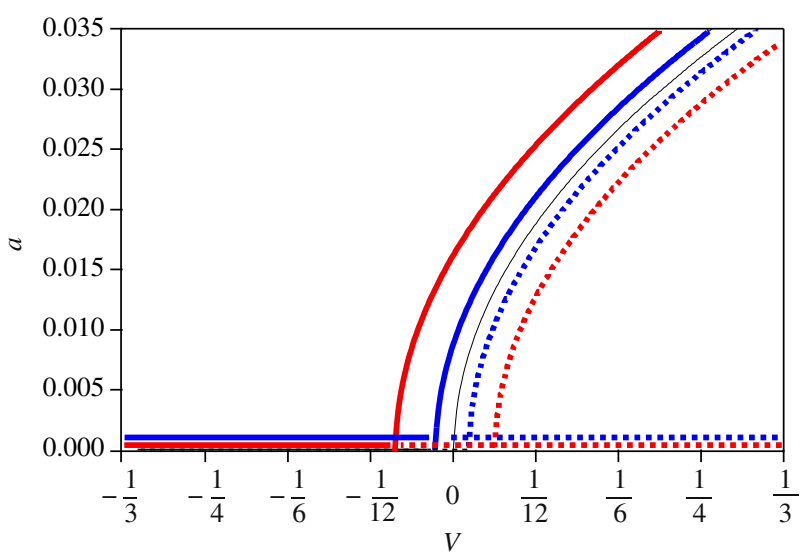

Fig. 7. Equilibrium branches in presence of turbulent wind when $u_{1}=0, \sigma \quad 0$. Black line: absence of turbulence; blue line: $u_{2}=0.033$; red line: $u_{2}=0.1$. Continuous line: stable; dashed line: unstable. (For interpretation of the references to color in this figure legend, the reader is referred to the web version of this article.)

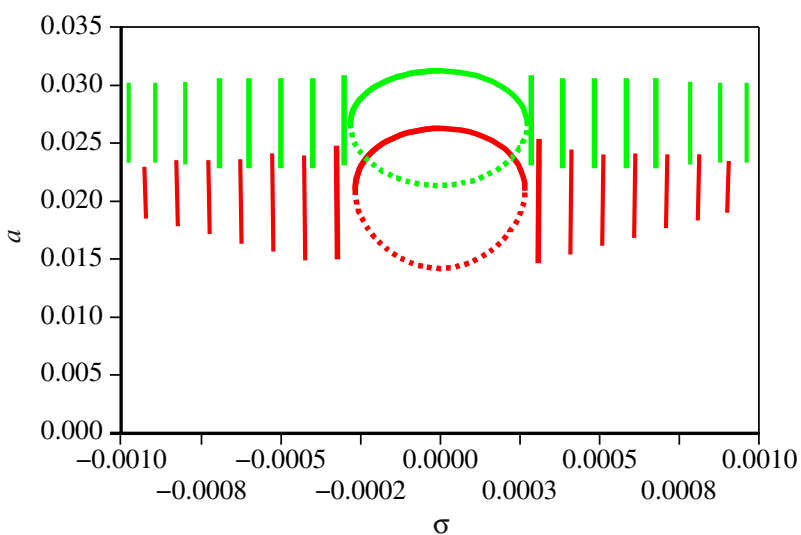

Fig. 8. Equilibrium branches in presence of turbulent wind when $u_{1}=0, u_{2}=0.10$. Red line: $V=0.10$; green line: $V=0.167$. Continuous line: stable; dashed line: unstable. (For interpretation of the references to color in this figure legend, the reader is referred to the web version of this article.)

In Fig. 8, plots of the amplitudes for fixed $V=0.10$ and 0.167 and varying $\sigma$ are shown. Outside the equilibrium branches, periodic solutions are found.

A three dimensional (qualitative) plot of the amplitude vs. $V$ and $\sigma$ is shown in Fig. 9. A tube is formed around the (not shown) perfect solution, emerging from the plane of the trivial solution $(a=0)$. Outside the tube, periodic solutions in terms of amplitude $a$ are found. 


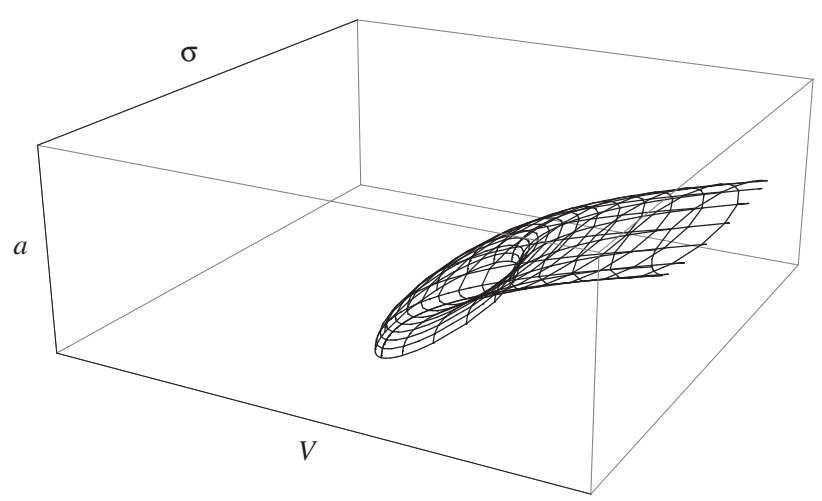

Fig. 9. Sketch of the equilibrium branch in presence of parametric excitation $\left(u_{1}=0, u_{2}=0.033\right)$.

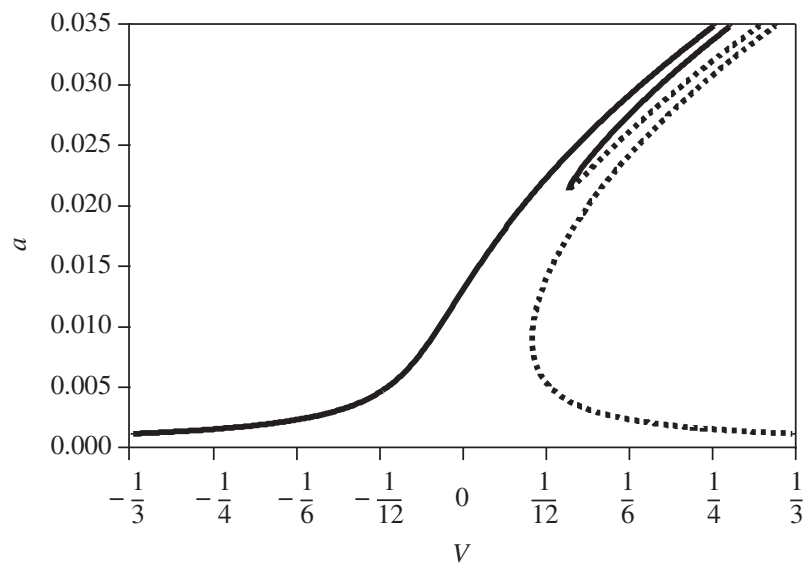

Fig. 10. Equilibrium branches in presence of turbulent wind when $u_{1}=u_{2}=0.033, \sigma \quad 0$. Continuous line: stable; dashed line: unstable.

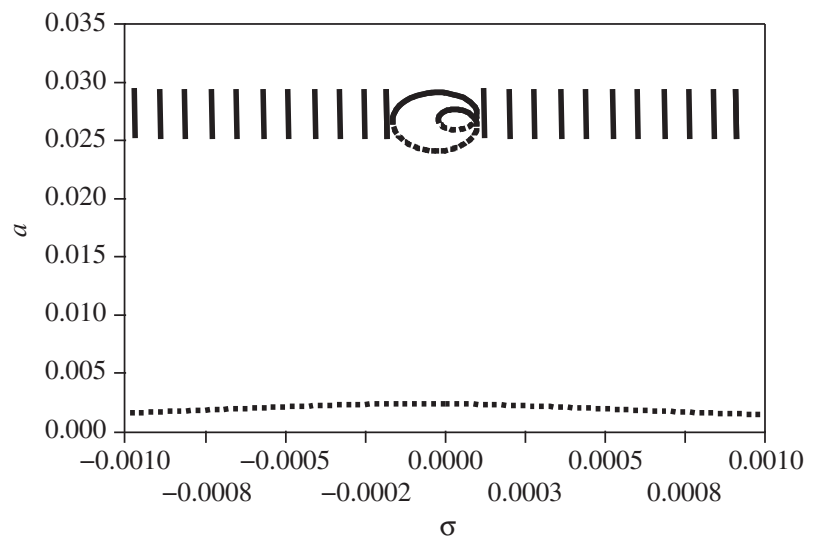

Fig. 11. Equilibrium branches in presence of turbulent wind when $u_{1}=u_{2}=0.033$. Continuous line: stable; dashed line: unstable.

When external and parametric excitations coexist (see Fig. 10, where $u_{1}=u_{2}=0.033$ ), a new branch adds to the scenario relevant to the externally excited system (Fig. 4), appearing inside the old branch. If a section (of a three dimensional plot not shown here) is taken at $V=0.167$, the plot of Fig. 11 is obtained, which highlights that the new branch is a loop of the existing branch. Again, periodic oscillations of the tower occur here; in particular, when the loop is present, two different amplitudes of oscillation are possible. Outside the tube, periodic solutions (with two periods) are found for the amplitude $a$, causing quasi periodic oscillations for the tower. 


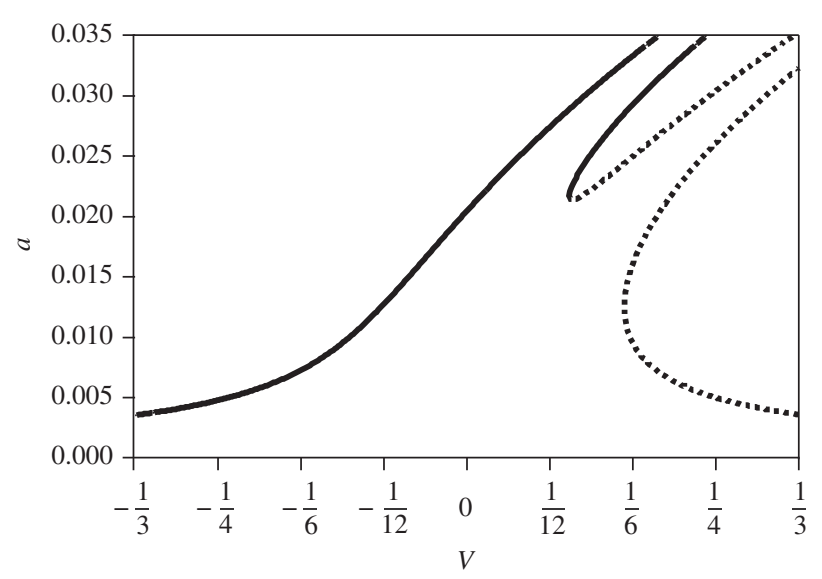

Fig. 12. Equilibrium branches in presence of turbulent wind when $u_{1}=u_{2}=0.10, \sigma \quad 0$. Continuous line: stable; dashed line: unstable.

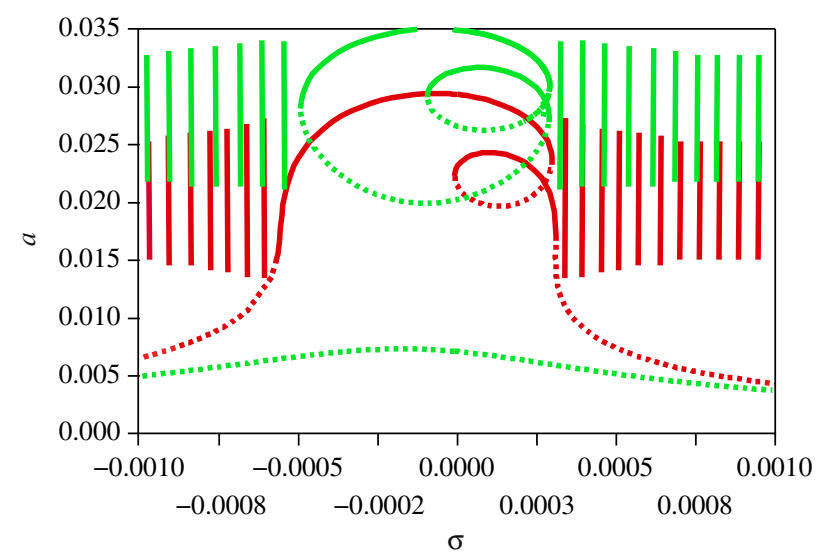

Fig. 13. Equilibrium branches in presence of turbulent wind when $u_{1}=u_{2}=0.10$. Red line: $V=0.110$; green line: $V=0.192$. Continuous line: stable; dashed line: unstable. (For interpretation of the references to color in this figure legend, the reader is referred to the web version of this article.)

If the strengths of the turbulent components are increased $\left(u_{1}=u_{2}=0.10\right)$, the equilibrium branches modify as shown in Fig. 12. The new branch producing the loop is even more prominent. Sections at $V=0.110$ and 0.192 are shown in Fig. 13. The red line is consistent with the results presented in [21].

\section{Conclusions}

In this paper a one dof nonlinear dynamical system, drawn by a Galerkin projection of a continuous structure, constituted by a rod subjected to turbulent wind, is considered. The system is subject to simultaneous self excitation, external and parametric excitations, the first due to the steady part of the aerodynamic force, the last two due to the turbulent part of the wind. The multiple scales method is used to obtain amplitude modulation equations, under the 1:1 resonance condition for the fundamental component of the turbulent wind. By taking the wind average speed and the fundamental frequency of the turbulent part as bifurcation parameters, the dynamical behavior of the system is studied. Different kinds of excitations are analyzed, in terms of equilibrium branches of the amplitudes. In particular, when the wind is non turbulent, a galloping curve is found. When the turbulence produces external excitation only, it works as an imperfection, slightly modifying the galloping curve, which acts as a backbone for the perturbed states. When the turbulence produces parametric excitation only, the galloping curve splits and translates in opposite directions, thus entailing reduction in the velocity of the incipient galloping. When the turbulence produces both external and parametric excitations, new branches occur, descending from loops.

\section{Acknowledgment}

This work was supported by the Italian Ministry of University (MIUR) through a PRIN co financed program. 
Appendix A. Coefficients of the equations of motion

The expressions of the coefficients of Eq. (11) are

$$
\begin{gathered}
\omega=\pi \frac{\sqrt{ } 3 E I}{h \ell \sqrt{ } m}, \quad c_{a}=\frac{\rho \mathcal{A}_{1} b h \ell \bar{U}_{c}}{2 \pi \sqrt{ } 3 E I m}, \quad b_{1}=c_{a}, \\
b_{2}=\frac{4 \rho \mathcal{A}_{2} b_{1} \ell}{3 \pi m}, \quad b_{31}=\frac{3 \pi \rho \mathcal{A}_{3} b \ell \sqrt{ } 3 E I}{8 h \bar{U}_{c} \sqrt{ } m^{3}}, \quad b_{32}=b_{31} \\
\eta_{1}=\frac{4 \rho \mathcal{A}_{0} b h^{2} \ell \bar{U}_{c}^{2}}{3 \pi^{3} E I}, \quad \eta_{2}=\frac{\eta_{1}}{2}
\end{gathered}
$$

and the dimensional critical wind velocity is

$$
\bar{U}_{c}=\frac{4 \pi \xi \sqrt{ } 3 E I m}{\rho b \mathcal{A}_{1} h \ell}
$$

Here, $\xi$ is the modal damping ratio, depending on both the external and internal dampings, according to Eq. (6) and the discretization adopted:

$$
\xi=\frac{\eta h^{2}}{24 E I} \omega+\frac{c}{2 m \omega}
$$

\section{Appendix B. Discussion on the approximation of the model}

Several approximations were introduced in modeling the original structure, namely: (i) transformation of the (discrete) shear type frame in a continuous one dimensional purely shear beam; (ii) reduction of the continuous model to a single dof system, via a modal truncation. The validity, from an engineering point of view, of these approximations are discussed here. First, the modal properties (eigenvalues and eigenfunctions) of the discrete and continuous models are compared; then, a numerical estimate of the contribution of the most important mode ignored in the analysis is carried out.

\section{B.1. Comparison of the modal properties}

The equations of motion of an $N$ stories planar shear type frame with story stiffness $k$ and story mass $m_{s}$ are

$$
\begin{aligned}
& k\left(v_{j-1}+2 v_{j} v_{j+1}\right)+m_{s} v_{j}=0, \quad j=1, \ldots, N \\
& v_{0}=0
\end{aligned}
$$

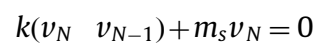

where $v_{j}(t)$ is the displacement of the $j$ th story and the index $j=0$ indicates the base level of the frame. The general solution for Eq. (B. $1_{1}$ ) has the form $v_{j}(t)=\phi_{j} \exp (i \omega t)$, with $\phi_{j}=\Lambda^{j}$ and $\Lambda$ a parameter to be evaluated. Substituting this expression for $v_{j}$ in Eq. (B. $\left.1_{1}\right)$, an algebraic equation in $\Lambda$ is obtained:

$$
\Lambda^{2} 2 \Lambda \cos \alpha+1=0
$$

where $\alpha$ is defined so that $\cos \alpha=1 \quad \omega^{2} m_{s} / 2 k$. Eq. (B.2) admits two roots: $\Lambda_{1,2}=\exp ( \pm i \alpha)$, therefore an expression for $\phi_{j}$ is obtained:

$$
\phi_{j}=c_{1} \cos (j \alpha)+c_{2} \sin (j \alpha)
$$

with $c_{1}$ and $c_{2}$ arbitrary constants. The application of the boundary conditions equation (B. $\left.1_{2,3}\right)$ provides the relation $c_{1}=0$ and the characteristic equation

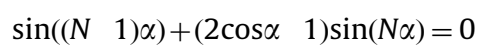

that can be solved numerically to get values for $\alpha$ and consequently for the natural frequency $\omega$. An asymptotic solution to Eq. (B.4) can, however, be obtained for large $N$. By ordering the variables as $N=\hat{N} / \varepsilon, \alpha=\varepsilon \hat{\alpha}$ with $\mathcal{O}(\hat{N})=\mathcal{O}(\hat{\alpha})=1$, and performing the limit for $\varepsilon \rightarrow 0$, the characteristic equation reduces to $\cos (N \alpha)=0$. This admits the solution $\alpha=\left(\begin{array}{ll}2 n & 1\end{array}\right) \pi / 2 N$, with $n=1,2, \ldots$, which confirms the assumption on the order of magnitude of $\alpha$, when $n \ll N$. By accounting for $1 \cos \alpha \simeq \alpha^{2} / 2$, the natural frequency of the $n$th mode is $\omega_{n}=\left(\left(\begin{array}{ll}2 n & 1) \pi / 2 N\end{array}\right) \sqrt{ } k / m_{s}\right.$ and its shape $\phi_{j, n}=\sin \left(j\left(\begin{array}{ll}2 n & 1\end{array}\right)\right.$ $\pi / 2 N)$. This asymptotic solution fully recovers the continuous solution relevant to the cantilever, namely $\omega_{n}=\left(\left(\begin{array}{ll}2 n & 1\end{array}\right) \pi / 2 \ell\right) \sqrt{ } G A / m, \phi_{n}(s)=\sin \left(\left(\begin{array}{ll}2 n & 1\end{array}\right) \pi s / 2 \ell\right)$, being $G A=12 E I / h^{2}, \ell=N h, m=m_{s} / h$ and $s$ being sampled at $s_{j}=h j$.

In the examined example, the number of stories is $N=9$, the story stiffness is $k=12 E I / h^{3}=21622204 \mathrm{~N} / \mathrm{m}$ and the story mass is $m_{s}=18947 \mathrm{~kg}$. The (exact) natural frequencies for the first three modes turn out to be $\omega_{1}=5.57 \mathrm{rad} / \mathrm{s}$, $\omega_{2}=16.58 \mathrm{rad} / \mathrm{s}, \omega_{3}=27.14 \mathrm{rad} / \mathrm{s}$, while the homogeneous model furnishes $\omega_{1}=5.89 \mathrm{rad} / \mathrm{s}, \omega_{2}=17.66 \mathrm{rad} / \mathrm{s}$, 


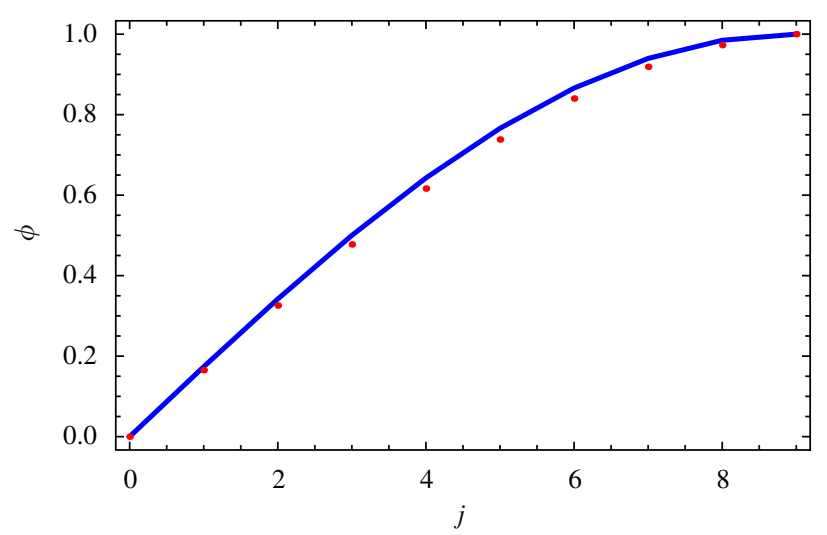

Fig. B1. First mode for the shear-type frame (red points) and for the homogeneous rod (blue line). (For interpretation of the references to color in this figure legend, the reader is referred to the web version of this article.)

$\omega_{3}=29.44 \mathrm{rad} / \mathrm{s}$, i.e. an error of 5 percent on the first mode. The first mode is shown in Fig. B1, in comparison with the first mode of the homogeneous model. They are in good agreement. The choice of the homogeneous model, rather than the discrete one, was suggested by the greater facility of the first in evaluating the modal forces via Galerkin projection.

\section{B.2. Estimate of the contribution of the second mode}

The previous analysis revealed the possible occurrence of internal resonance of 1:3 type between the first and second modes (being their frequency ratio equal to 2.976 in the discrete model and exactly to three in the homogeneous model). Therefore, in principle, the second mode should not be neglected in a Galerkin projection, but rather should be accounted for as active variable, in a center manifold perspective [3,29]. This is a well known problem, frequently encountered in dealing with continuous systems, which usually possess nearly commensurable frequencies (see e.g. [2, Chap. 7]). However, it must also be considered that, according to the Rayleigh model adopted for damping (Eq. (6)), while the external component ( $c$ in Eq. (6)) entails a decrement of the modal damping ratio $\xi_{n}$ with the mode number $n$, the internal component ( $\eta$ in Eq. (6)) instead increases such a ratio [30]. Indeed, for the shear cantilever rod under discussion it results as

$$
\xi_{2}=\frac{3 \eta h^{2}}{24 E I} \omega+\frac{c}{6 m \omega}
$$

to be compared with $\xi_{1} \equiv \xi$ of Eq. (A.3). On the other hand, the aerodynamic effects, which entail a negative damping, are unaffected by $n$, since the projection of uniformly distributed lift forces on the $n$th modal shape is proportional to the $n$th modal mass. All these circumstances suggest that, when the system is internally damped to a sufficient extent (as it happens in a true building), the second mode acts as a passive variable, i.e. it contributes as a small component to the overall response. In other words, it does not participate with its own frequency, although it is resonant, but rather it is forced by the (active) first mode.

To check this conjecture, a two dof Galerkin projection was performed, by assuming $v(s, t)=x(t) \phi_{1}(s)+y(t) \phi_{2}(s)$. By using the numerical values of the sample system, the following (non dimensional) coupled equations were drawn, instead of the single Eq. (22):

$$
\begin{aligned}
& x+(0.01 \quad 0.01(1+V+u(t))) \dot{x}+x+0.0155 \dot{x}^{2}+0.0119 \dot{y}^{2} \\
& 0.0062 \dot{x} \dot{y}+3.099(1 \quad V \quad u(t)) \dot{x}^{3} \quad 3.099(1 \quad V \quad u(t)) \dot{x}^{2} \dot{y} \\
& +6.119(1 \quad V \quad u(t)) \dot{x} \dot{y}^{2}=5.74 \times 10^{-5}(1+2 V+2 u(t)) \\
& y+(0.08 \quad 0.01(1+V+u(t))) \dot{y}+9 y \quad 0.0031 \dot{x}^{2}+0.0052 \dot{y}^{2} \\
& +0.0239 \dot{x} \dot{y} 1.033(1 \quad V \quad u(t)) \dot{x}^{3}+3.099(1 \quad V \quad u(t)) \dot{y}^{3} \\
& +6.199(1 \quad V \quad u(t)) \dot{x}^{2} \dot{y}=1.91 \times 10^{-5}(1+2 V+2 u(t))
\end{aligned}
$$

First of all, it is noticed that the non dimensional critical wind speed relevant to the second mode is $\bar{U}=0.08 / 0.01=8$, corresponding to $240 \mathrm{~m} / \mathrm{s}$. This entails that no Hopf bifurcations can involve such a mode in a realistic wind velocity range. Then, if one takes only the leading term in the $x$ expansion, i.e. $x(t)=a \cos (t)$, a (perfectly) resonant forcing term appears in the $y$ equation, generated by $\dot{x}^{3}(t)$ and proportional to $(1 / 4) a^{3} \cos (3 t)$. By taking the maximum value of $V=1 / 3$ considered in the numerical investigation, the amplitude of the corresponding $y$ response is

$$
|y(t)|=\frac{0.689 a^{3}}{4 * 0.066 * 3}=0.869 a^{3}
$$


It follows that $|y(t)| /|x(t)|=0.869 a^{2}$, with $a \simeq 0.03$ in the field of investigation. Therefore, the response of the system is essentially in the first mode.

\section{References}

[1] R. Clark, A Modern Course in Aeroelasticity, fourth ed., Kluwer Academic Publishers, Dordrecht, The Netherlands, 2004.

[2] A.H. Nayfeh, D.T. Mook, Nonlinear Oscillations, John Wiley, New York, 1979.

[3] A.H. Nayfeh, B. Balachandran, Applied Nonlinear Dynamics, John Wiley, New York, 1995.

[4] M.S. Soliman, Suppression of steady state bifurcations and premature fractal basin erosion in nonlinear systems subjected to combined external and parametric excitations, Chaos Solitons Fractals 4 (1994) 1871-1882.

[5] M. Belhaq, M. Houssni, Suppression of chaos in a nonlinear oscillator with parametric and external excitation, Nonlinear Anal. 30 (1997) $5147-5155$.

[6] N.E. Sanchez, A.H. Nayfeh, Global behavior of a biased non-linear oscillator under external and parametric excitations, J. Sound Vib. 207 (1997) 137-149.

[7] M. Belhaq, M. Houssni, Quasi-periodic oscillations, chaos and suppression of chaos in a nonlinear oscillator driven by parametric and external excitations, Nonlinear Dyn. 18 (1999) 1-24.

[8] W. Li, P. Xu, Bifurcation and chaos in a weakly nonlinear system subjected to combined parametric and external excitation, Acta Math. Appl. Sin. Engl. Ser. 18 (2002) 501-512.

[9] S.B. Yamgoue, T.C. Kofane, Chaotic responses of a deformable system under parametric and external excitations, Chaos Solitons Fractals 17 (2003) 155-167.

[10] G. Cheng, J.W. Zu, A numerical study of a dry friction oscillator with parametric and external excitations, J. Sound Vib. 287 (2005) 329-342.

[11] A.F. El-Bassiouny, Nonlinear vibration of a post-buckled beam subjected to external and parametric excitations, Phys. Scr. 74 (2006) 39-54.

[12] L. Ruihong, X. Wei, L. Shuang, Chaos control and synchronization of the $\Phi^{6}$ - van der pol system driven by external and parametric excitations, Nonlinear Dyn. 53 (2008) 261-271.

[13] M.F. Dimentberg, Response of a non-linearly damped oscillator to combined periodic parametric and random external excitation, Int. J. Nonlinear Mech. 11 (1976) 83-87.

[14] N.C. Menh, Responses of weakly non-linear dynamical systems subjected to random parametric and external excitations, J. Sound Vib. 113 (1987) $1-8$.

[15] G.E. Young, R.J. Chang, Prediction of the response of non-linear oscillators under stochastic parametric and external excitations, Int. J. Nonlinear Mech. 22 (1987) 151-160.

[16] M. Dimentberg, Z. Hou, M. Noori, W. Zhang, Response of an sdof system to periodic external and parametric excitations with random phase modulations, J. Sound Vib. 192 (1996) 621-627.

[17] O.S. Lu, C.W.S. To, Principal resonance of a nonlinear system with two-frequency parametric and self-excitations, Nonlinear Dyn. 2 (1991) $419-444$.

[18] A.F. El-Bassiouny, Principal parametric resonances of non-linear mechanical system with two-frequency and self-excitations, Mech. Res. Commun. 32 (2005) 337-350.

[19] M.Y. Izrailovich, Parametric excitation of stable self-vibrations in a mechanical system, J. Mech. Manuf. Reliab. 37 (2008) 12-15.

[20] K. Szabelski, J. Warminski, Self-excited system vibrations with parametric and external excitations, J. Sound Vib. 187 (1995) $595-607$.

[21] K. Szabelski, J. Warminski, Parametric self-excited non-linear system vibrations analysis with inertial excitation, Int. J. Nonlinear Mech. 30 (1995) 179-189.

[22] M. Eissa, Y.A. Amer, Vibration control of a cantilever beam subject to both external and parametric excitation, Appl. Math.Comput. 152 (2004) 611-619.

[23] H. Abdelhafez, Resonance of a nonlinear forced system with two-frequency parametric and self-excitations, Math. Comput. Simulation 66 (2004) 69-83.

[24] J. Warminski, Synchronisation effects and chaos in Van der Pol-Mathieu oscillator, J. Theor. Appl. Mech. 39 (2001) 861-884.

[25] M. Abdel-Rohman, Effect of unsteady wind flow on galloping of tall prismatic structures, Nonlinear Dyn. 26 (2001) $231-252$.

[26] A. Luongo, A. Paolone, Multiple scale analysis for divergence-Hopf bifurcation of imperfect symmetric systems, J. Sound Vib. 218 (1998) $527-539$.

[27] A. Luongo, G. Rega, F. Vestroni, On nonlinear dynamics of planar shear indeformable beams, J. Appl. Mech. 53 (1986) 619-624.

[28] M. Novak, Aeroelastic galloping of prismatic bodies, J. Eng. Mech. 95 (EM1) (1969) 115-141.

[29] H. Troger, A. Steindl, Nonlinear Stability and Bifurcation Theory. An Introduction for Engineers and Applied Scientists, Springer-Verlag, Wien, 1991.

[30] R.W. Clough, J. Penzien, Dynamics of Structures, third ed., Comput. Struct., Inc., Berkeley, CA, USA, 2003. 